\section{A RETROSPECTIVE STUDY OF PACKED RED BLOOD CELL TRANSFUSION IN NECROTISING ENTEROCOLITIS}

B. Thyagarajan, A. Shirsalkar

Neonatal Intensive Care Unit, Queens Hospital, Romford, UK

Aims: We evaluated the role of packed red blood cell (PRBC) transfusion on the causation of necrotising enterocolitis (NEC) in neonates.

Methods: In a retrospective observational study (April 08-March 09), we compared neonates who developed NEC after PRBC transfusion (transfusion group) to neonates who developed NEC without blood transfusion (controls).

Results: Over the one year period, 20 neonates developed NEC (2/1000 live births). Gestation less than 30 weeks $(75 \%)$ and birth weight less than 1500 grams (93.3\%) were the most common risk factors. Nine (55.6\%) babies had received a recent PRBC transfusion. The gestational age of the transfusion group (27.5 weeks) was comparable to the control group ( 26.89 weeks). NEC developed at a mean post natal age of 42 days in the transfusion group against 19.2 days in controls. $50 \%$ of babies in the transfusion group were Black African ethnicity compared to $14.3 \%$ in the control group. Perforating NEC developed in $22.2 \%$ of babies in the transfusion group (42.8\% in the control group). An abnormal antenatal ultrasound scan (23.1\%), maternal smoking (25\%), antepartum haemorrhage $(21.4 \%)$, chorioamnionitis (33.3\%) and emergency caesarean section delivery $(40 \%)$ were moderately associated with NEC, but were not significantly different between the groups. There was a bimodal pattern of onset. Early symptoms developed within 72 hours (mean 2.6 days) of the packed red cell transfusion in five (55.6\%) babies and late symptoms developed at mean 10.6 days.

Conclusions: There is a close association between blood transfusion and NEC. Black African babies and older babies are at increased risk.

\section{NITRIC OXIDE AND LIPID PEROXIDATION \\ ARE INCREASED AND ASSOCIATED WITH DECREASED ANTIOXIDANT ENZYME ACTIVITIES IN CHILDREN WITH NOSOCOMIAL PNEUMONIA}

\author{
D. Dmytriiev, O. Katilov, K. Dmytriieva \\ Vinnitsa National Medical University, $V$ \\ innitsa, Ukraine
}

Background: Nitric oxide (NO), hydroxyl radical $\left(\mathrm{OH}^{*}\right)$, superoxide anion (O2-) and hydrogen peroxide $(\mathrm{H} 2 \mathrm{O} 2)$ are free-radicals released in oxidative stress. Superoxide dismutase (SOD), glutathione peroxidase (GSHPx) and catalase (CAT) are antioxidant enzymes, mediating defense against oxidative stress. Excess $\mathrm{NO}$ and/or defective antioxidants cause lipid peroxidation, cellular dysfunction and death. Nosocomial pneumonia (NP) the leading cause of death in children.

Methods: NO, lipid peroxidation and the catalytic activity of SOD, GSHPx and CAT were measured in a group of 12 patients with nosocomial pneumonia ( 5 boys, 7 girls; $8.02+/-3.70$ years) and compared with age- and sex-matched healthy control subjects without NP ( 7 boys, 7 girls; $8.42+/-3.40$ days).

Results: All patients with NP had significantly $(p<$ 0.001 ) higher plasma NO levels over control subjects (40.18+/- 8.81 vs. $20.22+/-3.19$ micromol/l). On the other hand, SOD and GSHPx activities were significantly lower in both RBCs and plasma of patients with NP than in control subjects (RBCsSOD, $3400.00+/-400.22$ vs. $5302.10+/-403.10$ $\mathrm{U} / \mathrm{g} \mathrm{Hb}, \mathrm{p}<0.001$; plasma-SOD, $500.01+/-50.00$ vs. $710.10+/-40.10 \mathrm{U} / g$ protein, $p<0.001$; RBCsGSHPx, $600.10+/-40.02$ vs. $702.10+/-40.12 \mathrm{U} / \mathrm{g}$ $\mathrm{Hb}, \mathrm{p}<0.001$; plasma-GSHPx, $90.20+/-10.10$ vs. $138.80+/-9.06 \mathrm{U} / \mathrm{g}$ protein, $\mathrm{p}<0.001$ ). In addition, plasma NO levels were negatively correlated with SOD and GSHPx activities.

Conclusions: This study demonstrated for the first time that NO, the most abundant free-radical in the body, might be implicated in the pathophysiology of NP. 